\section{Uncoupling neurogenic gene networks in the Drosophila embryo}

\author{
William A. Rogers, ${ }^{1,3}$ Yogesh Goyal, ${ }^{2,3}$ \\ Kei Yamaya, ${ }^{1}$ Stanislav Y. Shvartsman, ${ }^{2}$ \\ and Michael S. Levine ${ }^{1}$
}

\begin{abstract}
${ }^{1}$ Department of Molecular Biology, Lewis-Sigler Institute for Integrative Genomics, Princeton University, Princeton, New Jersey 08544, USA; ${ }^{2}$ Department of Chemical and Biological Engineering, Lewis-Sigler Institute for Integrative Genomics, Princeton University, Princeton, New Jersey 08544, USA
\end{abstract}

The EGF signaling pathway specifies neuronal identities in the Drosophila embryo by regulating developmental patterning genes such as intermediate neuroblasts defective (ind). EGFR is activated in the ventral midline and neurogenic ectoderm by the Spitz ligand, which is processed by the Rhomboid protease. CRISPR/Cas9 was used to delete defined rhomboid enhancers mediating expression at each site of Spitz processing. Surprisingly, the neurogenic ectoderm, not the ventral midline, was found to be the dominant source of EGF patterning activity. We suggest that Drosophila is undergoing an evolutionary transition in central nervous system (CNS)-organizing activity from the ventral midline to the neurogenic ectoderm.

Supplemental material is available for this article.

Received February 5, 2017; revised version accepted March 20, 2017.

Transcriptional control of ligand production is important for the spatiotemporal patterning of cell fate during embryogenesis (Marinić et al. 2013). Limiting components of signaling pathways, such as a ligand or ligand-processing enzyme, are often controlled by multiple enhancers mediating distinct patterns of expression. There are many examples of complex gene control, including the FGF ligands Thisbe and Branchless and the BMP ligands Dpp and Gbb (Housden and Perrimon 2014). However, the specific contributions of individual enhancers to any given signaling process remain unclear. Here we address this question during the patterning of the neurogenic ectoderm in the Drosophila embryo, which is controlled by two EGF ligands: Vein and Spitz.

Vein is secreted from its site of synthesis, whereas Spitz requires processing by the membrane-bound Rhomboid protease. Vein and Spitz induce the expression of regulatory genes required for the specification of ventral and lateral neurons (Mayer and Nüsslein-Volhard 1988; Rutledge et al. 1992; Raz and Shilo 1993; Schweitzer et al. 1995; Schnepp et al. 1996; Golembo et al. 1996). High levels of

[Keywords: Drosophila embryo; EGFR regulatory networks; rhomboid; intermediate neuroblasts defective; central nervous system]

${ }^{3}$ These authors contributed equally to this work.

Corresponding authors: ms12@princeton.edu, stas@princeton.edu

Article published online ahead of print. Article and publication date are online at http://www.genesdev.org/cgi/doi/10.1101/gad.297150.117.
EGF/ERK signaling trigger the expression of the ventral nervous system defective ( $v$ nd) determinant in ventral regions of the neurogenic ectoderm, while low levels activate intermediate neuroblasts defective (ind) in lateral regions (Ohlen and Doe 2000; Stathopoulos and Levine 2005; Ajuria et al. 2011; Lim et al. 2013, 2015).

Most previous studies on the establishment of this EGF signaling gradient have emphasized the paracrine effects of EGF ligands emanating from the ventral midline (Golembo et al. 1996; Chang et al. 2001; Rousso et al. 2010). However, both vein (vn) and rhomboid (rho) are also expressed in ventral regions of the presumptive neurogenic ectoderm ("lateral stripes") prior to the onset of gastrulation (Kosman et al. 1991; Ip et al. 1992; González-Crespo and Levine 1993; Markstein et al. 2004). It has not been possible to assess the relative contributions of the two sites of EGF synthesis, the ventral midline and lateral stripes, in the patterning of the central nervous system (CNS).

Past studies identified separate enhancers for the regulation of rho expression in lateral stripes and the ventral midline. Midline expression depends on two separate enhancers, the midline enhancer (MLE) and a putative shadow enhancer (SHA) (Kvon et al. 2014; Pearson and Crews 2014), which is consistent with the view that the midline source of the Vein and Spitz ligands is particularly important for the patterning of the CNS. The role of the lateral stripe pattern, which is controlled by a well-characterized neurogenic ectoderm enhancer (NEE), remains uncertain (Supplemental Table 1).

Here, we used newly developed CRISPR/Cas9 methods to uncouple EGF synthesis in lateral stripes and the ventral midline (Barrangou et al. 2007; Jinek et al. 2012; Gratz et al. 2013). Mutant embryos harboring deletions of defined rho enhancers were combined with vn mutants, since past studies have shown that Spitz and Vein function in a somewhat redundant fashion to activate vnd and ind expression in ventral and lateral regions of the developing ventral nerve cord (Skeath 1998; Lim et al. 2015). Spitz activity is fully abolished by the loss of rho activity, and, consequently, rho; vn double mutants exhibit a complete loss of ind expression due to the absence of both Vein and activated Spitz (Hong et al. 2008).

We were therefore able to obtain mutant embryos containing a midline-only source of EGF signaling by the targeted deletion of the rho NEE in $\mathrm{vn}^{-} / \mathrm{vn}^{-}$mutant embryos. These embryos exhibit incomplete patterns of ind expression, suggesting that the midline is not sufficient to pattern the CNS. Surprisingly, the reciprocal experiment, restricted processing of Spitz in lateral stripes, produces an essentially normal ind expression pattern. These findings underscore the importance of rho and vn lateral stripes in the formation of the EGF signaling gradient and patterning of the CNS. Genome-editing methods provide a powerful means for deleting defined enhancers within endogenous loci to uncouple the complex

(C) 2017 Rogers et al. This article is distributed exclusively by Cold Spring Harbor Laboratory Press for the first six months after the full-issue publication date (see http://genesdev.cshlp.org/site/misc/terms.xhtml). After six months, it is available under a Creative Commons License (Attribution-NonCommercial 4.0 International), as described at http://creativecommons.org/licenses/by-nc/4.0/. 
regulatory control of critical developmental patterning genes such as rho.

\section{Results and Discussion}

Differential activation of $v n d$ and ind in ventral and lateral regions of the neurogenic ectoderm is controlled by Spitz and Vein EGF ligands (Ohlen and Doe 2000; Stathopoulos and Levine 2005; Ajuria et al. 2011; Lim et al. 2013, 2015) emanating from ventral regions of the neurogenic ectoderm (summarized in Fig. 1A). We used CRISPR/ Cas9 genome-editing methods to determine the relative contributions of EGF signaling in lateral stripes (ventral neurogenic ectoderm) and the ventral midline by deleting defined rho enhancers: MLE, NEE, and SHA (summarized in Fig. 1B; Barrangou et al. 2007; Jinek et al. 2012; Gratz et al. 2013). The SHA and MLE mediate expression in the midline in response to the Sim activator, whereas the NEE activates rho in the presumptive neurogenic ectoderm (lateral stripes) in response to the maternal dorsal gradient.

Midline-specific EGF signaling was obtained by deleting the rho NEE. Mutant embryos exhibit a slight delay in the onset of ind expression but nonetheless grow to adulthood (Supplemental Fig. S1A, $\mathrm{B}^{\prime \prime}$ ). Similar patterns of expression were also observed in $\mathrm{rho}^{-} / \mathrm{rho}^{-}$mutant embryos (Skeath 1998; Hong et al. 2008; Lim et al. 2013) as well as mutants harboring different combinations of rho enhancer deletions (Supplemental Fig. S1). These observations are consistent with previous findings that Vein alone is sufficient for the normal induction of ind expression (Supplemental Fig. S1). In order to assess the individual contributions of $\mathrm{rho}^{+}$gene activity in the midline and neurogenic ectoderm, it was necessary to introduce rho NEE deficiency homozygotes into a $v n^{-} / v n^{-}$mutant background.

The resulting double mutants, rho $\Delta \mathrm{NEE} v n^{-} /$rho $\triangle \mathrm{NEE} ; v n^{-}$, completely lack both $r h o$ and $v n$ lateral stripes (e.g., Fig. 1F). The only source of EGF signaling arises from the midline-specific expression of rho and localized pro-

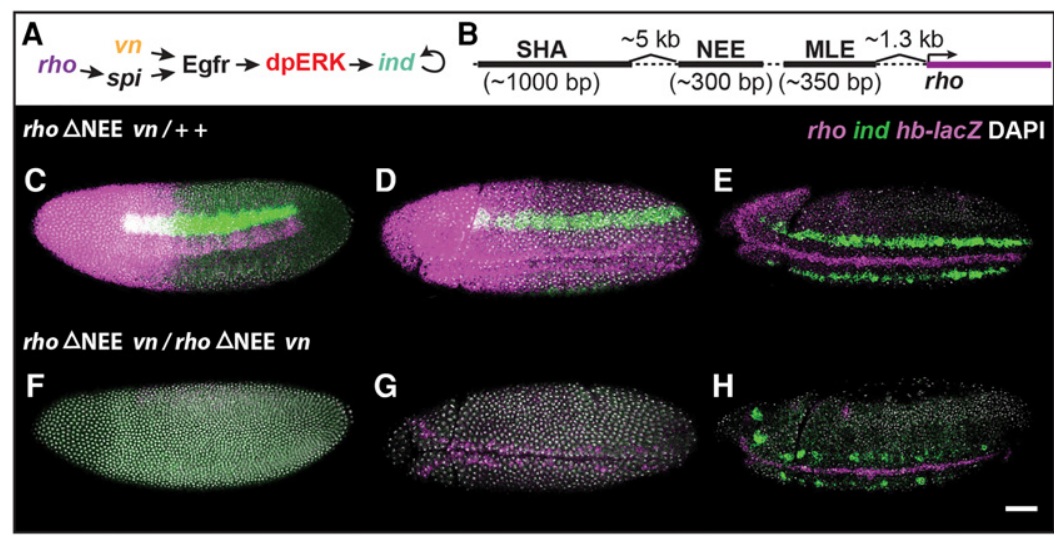

Figure 1. Late rho enhancers insufficient for normal ind expression. $(A)$ The EGFR pathway with arrows showing activation. $(B)$ The locations of known rho enhancers in relation to the genes. Black bars are the enhancers for the rho gene (purple bar). The dotted line indicates intergenic region. $(C-H)$ The rho $\triangle \mathrm{NEE}, \mathrm{vn}$ double mutant is orientated with anterior to the left and posterior to the right. Images from early NC14 $(C, F)$, gastrulation $(D, G)$, and germ band elongation $(E, H)$. $(C-E)$ Heterozygous mutants. $(F-H)$ Homozygous mutants. (Magenta lines) rho expression; (anterior magenta) $h b$-lacZ was used to visualize heterozygous mutants; (green lines) ind expression; (gray dots) DAPI. Bar, $50 \mu \mathrm{m}$. cessing of Spitz (Fig. 1E,H). Double-labeling methods using rho and ind RNA probes indicate a significant delay in the activation of ind (Fig. 1, cf. D and G). Moreover, the late ind expression pattern is incomplete and displays considerable variation between embryos (Fig. 1H). These observations suggest that the midline processing of the Spitz ligand is not sufficient for proper induction of ind expression and patterning of the nerve cord.

Most previous studies of EGF-mediated patterning of the CNS emphasized the role of the ventral midline as the source of ligand production (Golembo et al. 1996; Chang et al. 2001; Rousso et al. 2010). However, embryos with individual deletions of the MLE and SHA exhibit normal rho and ind expression patterns (Supplemental Fig. S1A-A",C-D" $\mathrm{D}^{\prime \prime}$. To more accurately assess the role of the midline, we produced embryos that selectively produce only lateral stripes of Spitz activity. This was achieved by creating sim;vn double-mutant embryos, which have transient lateral stripes of rho expression in the absence of midline activity (Fig. 2). Sim encodes a basic helix-loop-helix (bHLH)-PAS activator that functions as a "master regulator" of ventral midline differentiation. It is expressed in the presumptive midline prior to the onset of gastrulation, where it coordinates the expression of $>50$ different target genes, including rho and vein. $\mathrm{sim}^{-} /$ $\mathrm{sim}^{-}$mutant embryos display a severe loss of $r$ ho and $v n$ expression in the ventral midline (Crews et al. 1988; Thomas et al. 1988; Nambu et al. 1990, 1991; Chang et al. 2001; Kearney et al. 2004; Hong et al. 2013).

sim;Vn double mutants produce active Spitz ligands in lateral stripes due solely to the transient activity of the rho NEE. The initial induction of ind expression is virtually indistinguishable from that seen in normal embryos (e.g., Fig. 2A,D) despite the loss of midline targets of EGF signaling such as orthodenticle (Supplemental Fig. S2). We expected premature loss of ind expression due to the absence of sustained rho expression in the midline. However, ind expression was found to persist in advanced-stage embryos long after the loss of the transient rho lateral stripes (Fig. 2E,F). This pattern is significantly less variable than that seen when Spitz is processed solely in the midline (Fig. $1 \mathrm{H})$. We therefore conclude that transient lateral stripes of EGF signaling, rather than stable midline processing, provide the dominant source of EGF activity in the patterning of the Drosophila CNS (see below).

The maintenance of the ind expression pattern in sim;vn double mutants raises the possibility that autoregulatory mechanisms might be responsible for sustained expression at later stages of embryogenesis (Von Ohlen et al. 2007). For example, positive autofeedback is responsible for maintaining sim expression in the ventral midline of advanced-stage embryos (Kasai et al. 1992). To explore whether a similar mechanism might be used to regulate ind, we induced ectopic EGF signaling by placing the rho coding sequence under the control of ind regulatory sequences (Fig. 3).

Normally, rho lateral stripes straddle the ventral border of the ind expression pattern (e.g., Fig. 1C). The use of ind regulatory sequences causes a significant expansion of the rho expression pattern into more dorsal 


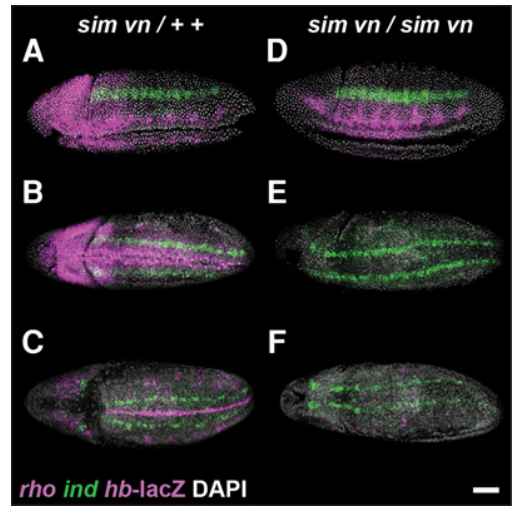

Figure 2. Early rho expression leads to stable late ind expression. The sim,vn heterozygous mutants $(A-C)$ and homozygous mutants $(D-F)$ are orientated with anterior to the left and posterior to the right. Images are from early $\mathrm{NCl} 4(A, D)$, gastrulation $(B, E)$, and germ band elongation $(C, F)$. (Magenta lines) rho expression. $(A-C)$ (Anterior magenta) $h b$-lacZ was used to visualize heterozygous mutants; (green lines) ind expression; (gray dots) DAPI. Bar, $50 \mu \mathrm{m}$.

regions of the neurogenic ectoderm. This expanded pattern leads to ectopic EGF signaling and dpERK activity in lateral regions of the developing nerve cord (Fig. 3, cf. $\mathrm{A}$ and $\mathrm{E}$ ). The initial ind expression pattern is considerably broader than the wild-type pattern (Fig. 3, cf. C and G) but nonetheless refines to a nearly normal pattern following gastrulation (Fig. 3, cf. D and H). This refinement occurs in spite of expanded EGF signaling, suggesting the occurrence of compensatory mechanisms of activation and repression of ind expression during development.

CRISPR/Cas9 genome-editing methods were used for the selective removal of defined developmental enhancers controlling the complex rho expression pattern during Drosophila embryogenesis (summarized in Supplemental Table 2). This approach has the potential to reveal new mechanisms of gene control. For example, the larval cuticles of rho $\triangle \mathrm{NEE}, \mathrm{vn}$ and sim;vn show no morphological defects (Supplemental Fig. S3), yet the removal of the 5' rho SHA and NEE appears to cause a delay in the onset of rho MLE activity in the ventral midline (Supplemental Fig. S4). The specific deletion of the rho NEE led to an incomplete and variable ind expression pattern, suggesting that the ventral midline may not be the central organizer of CNS patterning suggested by earlier studies (Golembo et al. 1996; Chang et al. 2001; Rousso et al. 2010). Instead, we showed that transient lateral stripes of EGF signaling are sufficient to induce ind expression, while subsequent compensatory mechanisms maintain this expression during embryogenesis.

The ventral midline is thought to be the ancestral mode of EGF signaling in the developing CNS of insects and other arthropods. For example, the ventral midline of the crustacean Parhyale plays a dominant role in the patterning of the CNS (Vargas-Vila et al. 2010). Disruption of $\mathrm{sim}^{+}$gene activity or ablation of midline cells causes severe patterning defects, whereas sim mutants in Drosophila exhibit essentially normal patterning of the nerve cord (except for the differentiation of mesectoderm cells arising from the midline) (Vargas-Vila et al. 2010; Lynch and Roth 2011). We propose that Drosophila represents a transitional patterning system in which the ancestral midline mode of EGF signaling is replaced by lateral stripes (Fig. 4). Rhomboid does not exhibit obvious lateral stripes of expression in other insects such as flour beetles and mosquitoes. This novel pattern of expression appears to coincide with the duplication of rhomboid genes in the Drosophilids (Crocker et al. 2010; Rousso et al. 2010).

Drosophila appears to use two distinct gene regulatory networks for deploying EGF signaling during embryogenesis. The expression of EGF signaling components in the ventral midline depends on the Sim activator, which in turn is induced by Notch signaling (Zinzen et al. 2006). In contrast, lateral stripes of $r h o$ and $v n$ expression depend on dorsal and bHLH activators, including proneural determinants such as Daughterless and Achaete/Scute (González-Crespo and Levine 1993). The latter mode of EGF signaling is dominant in Drosophila, but the retention of midline patterning activity might render the system robust to genetic and environmental variation. This view of network evolution, the co-option of novelty while retaining ancestral mechanisms, might be a common property of animal development.

\section{Materials and methods \\ CRISPR-Cas9 (Supplemental Tables 3-5)}

Guide RNA ( $g R N A$ ) The protocol was followed based on the publication by the O'Connor-Giles laboratory, and the unique PAM recognition sites were designed with the CRISPR optimal target finder (http://tools. flycrispr.molbio.wisc.edu/targetFinder; Gratz et al. 2014). The two unique PAM recognition sites were inserted into individual pU6b-gRNA vectors by BbsI. PAM sites were verified in the injection line. The pU6b-gRNA was obtained from the Perrimon laboratory (Supplemental Material; Ren et al. 2013).

Donor vectors The p2xattP-dsRed donor vector was modified from the O'Connor-Giles laboratory pHD-DsRed-attP vector by the addition of an inverted attP site $3^{\prime}$ of the dsRed cassette (Supplemental Material). An $\sim 1-\mathrm{kb} 5$ ' homology arm was inserted with NheI and SacII. An $\sim 1-\mathrm{kb} 3^{\prime}$ homology arm was inserted with SbfI and AscI (modified MCS). The GFP donor vector (gift from Thomas Gregor's laboratory) used XhoI/AscI and AgeI/NotI for the $5^{\prime}$ and $3^{\prime}$ homology arms, respectively. CRISPR vectors (donor vector and two unique guide vectors) were injected into nos-Cas9 (y sc v; (nos-Cas9) $a$ attP40/CyO [Bestgene: TH00788.N]). The double enhancer deletions were produced by injecting previous dsRed CRISPR fly lines with the GFP donor vector (Supplemental Material), two unique

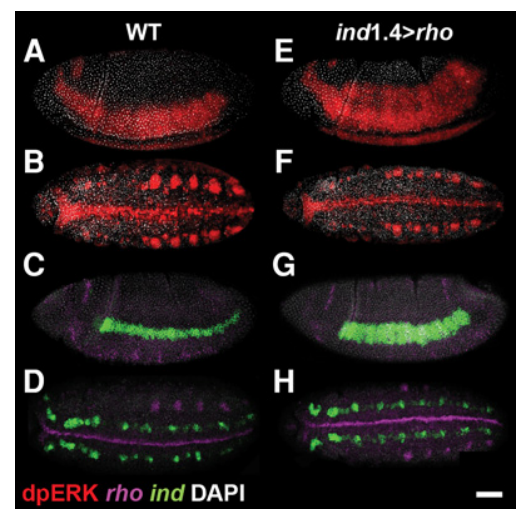

Figure 3. Early ectopic ind expression compensates for normal late expression. $(A-D)$ Wild type (WT). $(E-H)$ Homozygous rho overexpression. Images were taken at gastrulation $(A, E, C, G)$ and germ band extension $(B, F, D, H)$. All images are anterior to posterior. (Red lines) dpERK expression; (purple lines) rho expression; (green lines) ind expression; (gray dots) DAPI. Bar, $50 \mu \mathrm{m}$. 


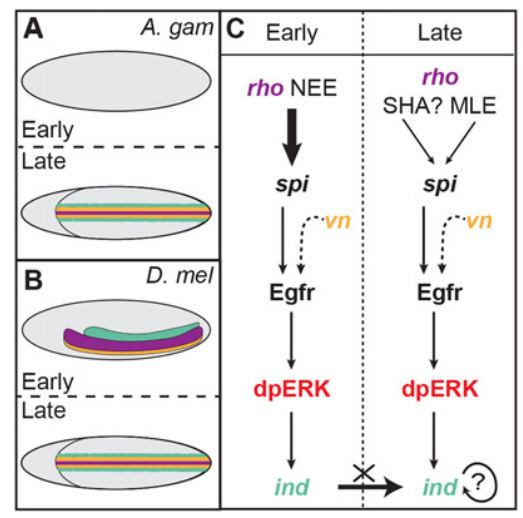

Figure 4. The ancestral rho MLE shifts its role to NEE for midline ind expression. (A) Mosquitos (Anopheles gambiae) rely primarily on the midline expression of rho for late ind expression. (B) Fruit fly (Drosophila melanogaster) ind regulation has a unique lateral expression followed by the ancestral late stage mediated mostly by rho. $(C)$ A. gambiae has no lateral regulation of ind $(\mathrm{X})$ and roles of rho SHA, and ind autoregulation are unknown (?). D. melanogaster seems to have acquired a major role for the rho NEE (large arrow) for regulating ind (arrow) while minimizing the role of the midline rho MLE and SHA (small arrows). (Purple) rho; (orange) vn; (green) ind.

guide vectors, and the nos-Cas9 plasmid. All lines were PCR-validated and imaged. All injections were performed by Bestgene. Primers were made by IDT.

\section{Immunostaining and fluorescent in situ hybridization (FISH)}

Immunostaining and FISH protocols were performed as described elsewhere (Lim et al. 2015). Sheep anti-digoxigenin (DIG; 1:125; Roche), mouse anti-biotin (1:125; Jackson ImmunoResearch), and rabbit anti-dpERK (1:100; CST4370) were used as primary antibodies. DAPI (1:10,000; Molecular Probes) was used to stain for nuclei, and Alexa fluor conjugates (1:500; Invitrogen) were used as secondary antibodies.

\section{Fly recombination}

;; vn,sim The $v n^{\mathrm{L} 6} / \mathrm{TM} 3, h b$-lacZ was crossed to sp/+;sim² $/ \mathrm{TM} 3, \mathrm{hb}$-lacZ. The virgin female vn/sim flies were crossed to dsRed/TM3,hb-lacZ. Recombination was assayed by crossing individual recombinant males to virgins of each mutant line. Validated lines exhibited TM3 from the assay $(n$ $=137)$.

;; Vn,rho $\triangle N E E$ The $v n^{\mathrm{L} 6} / \mathrm{TM} 3, h b$-lacZ line was crossed to $r$ ho $\triangle \mathrm{NEE} /$ TM3,hb-lacZ. The female virgin $v n / r h o \quad \Delta$ NEE flies were crossed to TM3,hb-lacZ. Individual males were tested for recombination by crossing to virgin vn mutants. Validated lines exhibited both TM3 and dsRed from the assays $(n=63)$.

\section{Microscopy and image processing}

Fluorescent imaging was performed on Nikon A1-RS scanning confocal (Fig. 1, 2) and Zeiss 880 (Fig. 3) microscopes with a 20× objective. For pairwise comparisons of wild-type and mutant backgrounds, embryos were collected, stained, and imaged together under the same experimental conditions. Broken embryos, embryos with intact vitelline membrane, or embryos undergoing mitosis were excluded from the analysis. The contrast and brightness for images were adjusted in ImageJ relative to the wildtype images for the final figure panels, thus allowing quantitative comparisons between two sets of images.

\section{Acknowledgments}

We thank members of the Levine and Shvartsman laboratories for their invaluable comments and advice. We also thank Hongtao Chen and Thomas Gregor for providing many of the CRISPR/Cas9 plasmids used in our experiments, and Gary Laevsky and Evangelos G. Gatzogiannis for their expert help with imaging. W.A.R. was supported by a National Institutes of Health (NIH) grant to M.S.L. (R35 GM118147). Y.G. and K.Y. were supported by an NIH grant to S.Y.S. (R01 GM086537). W.A.R., Y.G., S.Y.S., and M.S.L. conceived and designed the project; W.A.R. designed the CRISPR/Cas9 mutant flies; Y.G., W.A.R., and K.Y. performed the imaging experiments; W.A.R. and Y.G. analyzed the results; and W.A.R., Y.G., S.Y. S., and M.S.L. wrote the manuscript.

\section{References}

Ajuria L, Nieva C, Winkler C, Kuo D, Samper N, Andreu MJ, Helman A, González-Crespo S, Paroush Z, Courey AJ, et al. 2011. Capicua DNA-binding sites are general response elements for RTK signaling in Drosophila. Development 924: 915-924.

Barrangou R, Fremaux C, Deveau H, Richards M, Boyaval P, Moineau S, Romero DA, Horvath P. 2007. CRISPR provides acquired resistance against viruses in prokaryotes. Science 315: 1709-1712.

Chang J, Kim IO, Ahn JS, Kim SH. 2001. The CNS midline cells control the spitz class and Egfr signaling genes to establish the proper cell fate of the Drosophila ventral neuroectoderm. Int I Dev Biol 45: 715-724.

Crews ST, Thomas JB, Goodman CS. 1988. The Drosophila single-minded gene encodes a nuclear protein with sequence similarity to the per gene product. Cell 52: 143-151.

Crocker J, Potter N, Erives A. 2010. Dynamic evolution of precise regulatory encodings creates the clustered site signature of enhancers. Nat Commun 1: 99.

Golembo M, Raz E, Shilo BZ. 1996. The Drosophila embryonic midline is the site of Spitz processing, and induces activation of the EGF receptor in the ventral ectoderm. Development 122: 3363-3370.

González-Crespo S, Levine M. 1993. Interactions between dorsal and helix-loop-helix proteins initiate the differentiation of the embryonic mesoderm and neuroectoderm in Drosophila. Genes Dev 7: 1703-1713.

Gratz SJ, Cummings AM, Nguyen JN, Hamm DC, Donohue LK, Harrison MM, Wildonger J, O'Connor-Giles KM. 2013. Genome engineering of Drosophila with the CRISPR RNA-guided Cas9 nuclease. Genetics 194: 1029-1035.

Gratz SJ, Ukken FP, Rubinstein CD, Thiede G, Donohue LK, Cummings AM, O'Connor-Giles KM. 2014. Highly specific and efficient CRISPR/Cas9-catalyzed homology-directed repair in Drosophila. Genetics 196: 961-971.

Hong J-W, Hendrix DA, Papatsenko D, Levine MS. 2008. How the dorsal gradient works: insights from postgenome technologies. Proc Natl Acad Sci 105: 20072-20076.

Hong J-W, Park KW, Levine MS. 2013. Temporal regulation of single-minded target genes in the ventral midline of the Drosophila central nervous system. Dev Biol 380: 335-343.

Housden BE, Perrimon N. 2014. Spatial and temporal organization of signaling pathways. Trends Biochem Sci 39: 457-464.

Ip YT, Park RE, Kosman D, Bier E, Levine M. 1992. The dorsal gradient morphogen regulates stripes of rhomboid expression in the presumptive neuroectoderm of the Drosophila embryo. Genes Dev 6: 1728-1739.

Jinek M, Chylinski K, Fonfara I, Hauer M, Doudna JA, Charpentier E. 2012. A programmable dual-RNA-guided DNA endonuclease in adaptive bacterial immunity. Science 337: 816-821.

Kasai Y, Nambu JR, Lieberman PM, Crews ST. 1992. Dorsal-ventral patterning in Drosophila: DNA binding of snail protein to the singleminded gene. Proc Natl Acad Sci 89: 3414-3418.

Kearney JB, Wheeler SR, Estes P, Parente B, Crews ST. 2004. Gene expression profiling of the developing Drosophila CNS midline cells. Dev Biol 275: 473-492.

Kosman D, Ip YT, Levine M, Arora K. 1991. Establishment of the mesoderm-neuroectoderm boundary in the Drosophila embryo. Science 254: 118-122. 
Kvon EZ, Kazmar T, Stampfel G, Yáñez-Cuna JO, Pagani M, Schernhuber K, Dickson BJ, Stark A. 2014. Genome-scale functional characterization of Drosophila developmental enhancers in vivo. Nature 517: 91-95.

Lim B, Samper N, Lu H, Rushlow C, Jiménez G, Shvartsman SY. 2013. Kinetics of gene derepression by ERK signaling. Proc Natl Acad Sci 110: 10330-10335.

Lim B, Dsilva CJ, Levario TJ, Lu H, Schüpbach T, Kevrekidis IG, Shvartsman SY. 2015. Dynamics of inductive ERK signaling in the Drosophila embryo. Curr Biol 25: 1784-1790.

Lynch JA, Roth S. 2011. The evolution of dorsal-ventral patterning mechanisms in insects. Genes Dev 25: 107-118.

Marinić M, Aktas T, Ruf S, Spitz F. 2013. An integrated holo-enhancer unit defines tissue and gene specificity of the Fgf8 regulatory landscape. Dev Cell 24: 530-542.

Markstein M, Zinzen R, Markstein P, Yee K, Erives A, Stathopoulos A, Levine M. 2004. A regulatory code for neurogenic gene expression in the Drosophila embryo. Development 131: 2387-2394.

Mayer U, Nüsslein-Volhard C. 1988. A group of genes required for pattern formation in the ventral ectoderm of the Drosophila embryo. Genes Dev 2: 1496-1511

Nambu JR, Franks RG, Hu S, Crews ST. 1990. The single-minded gene of Drosophila is required for the expression of genes important for the development of CNS midline cells. Cell 63: 63-75.

Nambu JR, Lewis JO, Wharton KA, Crews ST. 1991. The Drosophila single-minded gene encodes a helix-loop-helix protein that acts as a master regulator of CNS midline development. Cell 67: 1157-1167.

Pearson JC, Crews ST. 2014. Enhancer diversity and the control of a simple pattern of Drosophila CNS midline cell expression. Dev Biol 392: 466-482.

Raz E, Shilo BZ. 1993. Establishment of ventral cell fates in the Drosophila embryonic ectoderm requires DER, the EGF receptor homolog. Genes Dev 7: 1937-1948.

Ren X, Sun I, Housden BE, Hu Y, Roesel C, Lin S, Liu L-P, Yang Z, Mao D, Sun L, et al. 2013. Optimized gene editing technology for Drosophila melanogaster using germ line-specific Cas9. Proc Nat1 Acad Sci 110: 19012-19017.
Rousso T, Lynch J, Yogev S, Roth S, Schejter ED, Shilo B. 2010. Generation of distinct signaling modes via diversification of the Egfr ligand-processing cassette. Development 137: 3427-3437.

Rutledge BJ, Zhang K, Bier E, Jan YN, Perrimon N. 1992. The Drosophila spitz gene encodes a putative EGF-like growth factor involved in dorsal-ventral axis formation and neurogenesis. Genes Dev 6: 15031517.

Schnepp B, Grumbling G, Donaldson T, Simcox A. 1996. Vein is a novel component in the Drosophila epidermal growth factor receptor pathway with similarity to the neuregulins. Genes Dev 10: 23022313.

Schweitzer R, Shaharabany M, Seger R. 1995. Secreted Spitz triggers the DER signaling pathway and is a limiting component in embryonic ventral ectoderm determination. Genes Dev 9: 1518-1529.

Skeath JB. 1998. The Drosophila EGF receptor controls the formation and specification of neuroblasts along the dorsal-ventral axis of the Drosophila embryo. Development 125: 3301-3312.

Stathopoulos A, Levine M. 2005. Localized repressors delineate the neurogenic ectoderm in the early Drosophila embryo. Dev Biol 280: 482-493.

Thomas JB, Crews ST, Goodman CS. 1988. Molecular genetics of the single-minded locus: a gene involved in the development of the Drosophila nervous system. Cell 52: 133-141.

Vargas-Vila MA, Hannibal RL, Parchem RJ, Liu PZ, Patel NH. 2010. A prominent requirement for single-minded and the ventral midline in patterning the dorsoventral axis of the crustacean Parhyale hawaiensis. Development 137: 3469-3476.

Von Ohlen T, Doe CQ. 2000. Convergence of dorsal, Dpp, and Egfr signaling pathways subdivides the Drosophila neuroectoderm into three dorsal-ventral columns. Dev Biol 224: 362-372.

Von Ohlen TL, Harvey C, Panda M. 2007. Identification of an upstream regulatory element reveals a novel requirement for Ind activity in maintaining ind expression. Mech Dev 124: 230-236.

Zinzen RP, Cande J, Ronshaugen M, Papatsenko D, Levine M. 2006. Evolution of the ventral midline in insect embryos. Dev Cell 11: 895-902. 


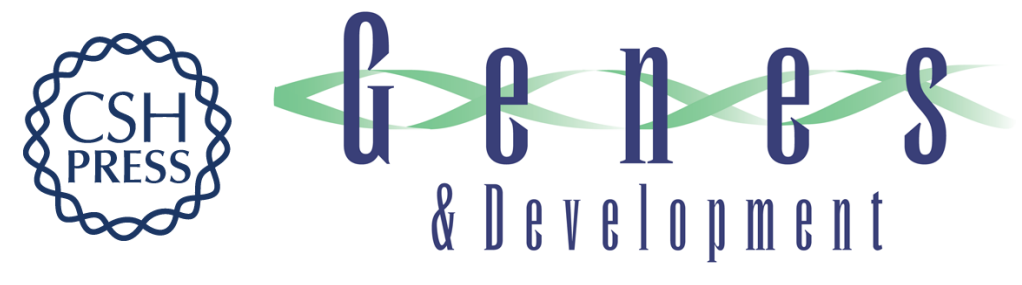

\title{
Uncoupling neurogenic gene networks in the Drosophila embryo
}

William A. Rogers, Yogesh Goyal, Kei Yamaya, et al.

Genes Dev. 2017, 31: originally published online April 20, 2017

Access the most recent version at doi:10.1101/gad.297150.117

\author{
Supplemental http://genesdev.cshlp.org/content/suppl/2017/04/20/gad.297150.117.DC1 \\ Material \\ Related Content Creating cell type-specific mutants by enhancer mutagenesis \\ Stephen Crews \\ Genes Dev. April , 2017 31: 629-631
}

References This article cites 40 articles, 22 of which can be accessed free at: http://genesdev.cshlp.org/content/31/7/634.full.html\#ref-list-1

Articles cited in: http://genesdev.cshlp.org/content/31/7/634.full.html\#related-urls

Creative This article is distributed exclusively by Cold Spring Harbor Laboratory Press for the first Commons six months after the full-issue publication date (see

License http://genesdev.cshlp.org/site/misc/terms.xhtml). After six months, it is available under a Creative Commons License (Attribution-NonCommercial 4.0 International), as described at http://creativecommons.org/licenses/by-nc/4.0/.

Email Alerting Receive free email alerts when new articles cite this article - sign up in the box at the top Service right corner of the article or click here.

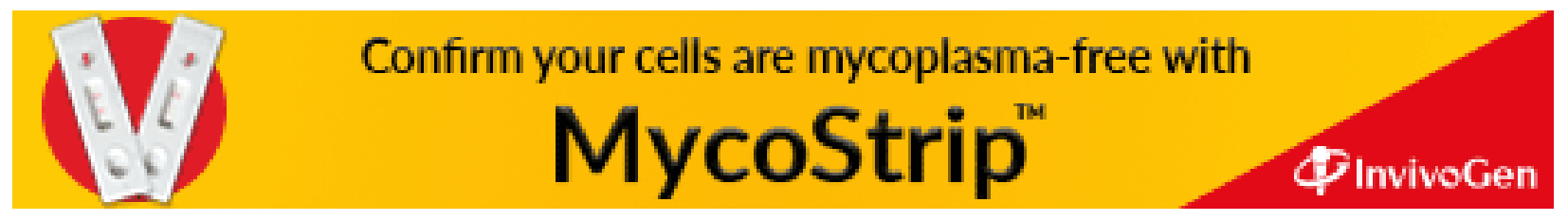

